\title{
Correction to: Backbone and side-chain chemical shift assignments of the kringle domain of human receptor tyrosine kinase-like orphan receptor 1 (ROR1)
}

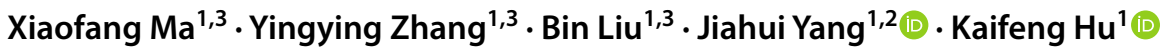

Published online: 28 June 2018

(c) Springer Nature B.V. 2018

\section{Correction to: \\ Biomolecular NMR Assignments (2018) 12:145-148 \\ https://doi.org/10.1007/s12104-017-9797-9}

The authors would like to include an additional institution to their affiliation which was initially not included in the original publication of the article. The affiliation is provided in this correction.

The original article can be found online at https://doi.org/10.1007/ s12104-017-9797-9.

Jiahui Yang

yjhsmmu@hotmail.com

$\triangle$ Kaifeng Hu

kaifenghu@mail.kib.ac.cn

1 State Key Laboratory of Phytochemistry and Plant

Resources in West China, Kunming Institute of Botany,

Chinese Academy of Sciences, Kunming, Yunnan 650201,

China

2 School of Basic Medicine, Chengdu University of TCM, Chengdu, Sichuan 611137, China

3 University of the Chinese Academy of Sciences, Beijing 100049, China 